\title{
Rezension zu Schlücker, Barbara (2014): Grammatik im Lexikon. Adjektiv-Nomen-Verbindungen im Deutschen und Niederländi- schen. Berlin/Boston: De Gruyter. (= Linguistische Arbeiten 553)
}

\author{
Clemens Knobloch (Siegen)
}

Adjektiv-Substantiv-Komposita, in denen (traditionell-grammatisch gesprochen) ein Adjektiv als Bestimmungswort und ein Substantiv als Grundwort fungiert, bilden im Deutschen einen eigentümlichen Verbund. Anders als in den konversen Konstruktionen aus substantivischem Bestimmungswort und adjektivischem Grundwort (mausgrau, himmelblau, knallrot, mausetot, strohdumm, stockfinster, grasgrün, haushoch, ...), in denen buchstäblich nur die Operationen „Vergleich“ und „Gradierung“ gehen, und einer zweiten Gruppe mit Bestimmungswörtern, die zusätzliche Argumente des Adjektivs sind, Rektionskomposita gewissermaßen (blutarm, geldgierig, liebesbedürftig, drogensüchtig, machtgeil, schlangengleich...), sind die Konstruktionen im Fokus der Untersuchung von Barbara Schlücker empirisch auf den ersten Blick mächtig heterogen. Es geht um Bildungen wie Weißwein, Grünkohl, Blaukraut, Schwarzbrot, Festgeld, Blödmann, Gutmensch, Fertigpizza, Schöngeist, Altkanzler, Faultier, Blindfisch, Leergut, Rundholz, Hochhaus, Niedrigwasser etc. Für gewöhnlich bilden diese Komposita ein eher randliches, selten gründlich erleuchtetes Kapitel in den Lehr- und Handbüchern der Wortbildung. Barbara Schlücker geht von vornherein anders an die Sache heran: Sie kontrastiert die AdjektivSubstantiv- Komposita (A+N-Komposita) systematisch mit ihren phrasalen Gegenstücken, mit den entsprechenden Adjektiv-Substantiv-Phrasen (A+N-Phrasen) mit dem Ziel, systematische Unterschiede zwischen wortinterner Modifikation und syntaktischer Modifikation zu erheben.

Kompliziert ist die Ausgangslage dadurch, dass es durchaus auch lexikalische A+N-Phrasen gibt: saure Sahne, blauer Fleck, kaltes Büfett, rote Beete etc., die in ihrer klassifikatorischen, konzeptmodifizierenden Bedeutung dem default-Typ der A+N-Komposita durchaus ähneln. Weiterhin gibt es auch eine diachrone Komplikation (cf. Schlücker 2014: 24): Historische Relikte aus der Zeit vor der obligatorischen Flexion von Adjektivattributen, die teilweise (ruhig Blut, täglich Brot) feste A $+\mathrm{N}-\mathrm{Phrasen}$ geworden sind, teilweise aber auch, wenn dies durch ihre morphologische Einfachheit möglich war, als Komposita reinterpretiert wurden (Junggeselle, Edelmann, Neujahr), wie Paul (1920: 337f.) schon gezeigt hat, während einige sogar trotz flexionaler „Reste“ univerbiert worden sind (Hohepriester, Langeweile, Blindekuh). 
Das Buch hat folgenden Aufbau: Auf das programmatische Einleitungskapitel folgt Kapitel 2 über „Morphosyntaktische Eigenschaften von deutschen A+N-Komposita“. Hier geht es zunächst um die prosodische Opposition zwischen A+N-Komposita (Hauptbetonung auf dem Modifikator) und A+N-Phrasen mit Hauptbetonung auf dem Kopf. Letzterem würde wohl ein Gesprochene-Sprache-Forscher widersprechen (und darauf bestehen, dass im A+N-Syntagma die Tonverhältnisse pragmatisch frei bleiben, dass bei restriktiver und kontrastiver Verwendung das Adjektiv den Starkton hat etc.). Bedeutsamer sind die morphologischen Restriktionen: Nur monomorphemische Adjektive sind in den Komposita zulässig (cf. Schlücker 2014: 29f.). Superlative kommen häufig vor (Höchstleistung, Schwerstarbeit etc.), Komparative gar nicht. Griechisch-lateinische Relationsadjektive auf - al, -iv, -är, -ar bilden die einzige Gruppe morphologisch komplexer Adjektive, die zulässig sind (Sozialarbeit, Suggestivfrage, Primärtumor, Solarzelle). Die berühmten Fertig-Komposita (Fertighaus, Fertigpizza etc.) gehen nur, weil fertig synchron nicht als morphologisch komplex interpretiert wird (ähnlich: niedrig-, flüssig-; cf. ibd.: 31). Darauf komme ich zurück.

Kapitel 3 ist der Kontrastierung von syntaktischer und morphologischer Modifikation gewidmet. Ausgehend von der älteren Theorie der „semantischen Spezialisierung“ von Komposita gegenüber den Syntagmen (nicht jeder schnelle Zug ist auch ein Schnellzug) entwickelt die Autorin ihre These von der „grundlegenden klassifikatorischen Bedeutung“ von A+N-Komposita (ebd.: 44), der Modifikator identifiziert dabei die Subklasse. Hierin gleichen die A+NKomposita der N+N-Komposition. Semantische Spezialisierung hingegen ist an Lexikalisierungsprozesse gebunden und findet sich auch bei Syntagmen. Die Voraussetzungen, die für eine nicht-klassifikatorische Lesart von A+N-Komposita gegeben sein müssen, diskutiert das folgende Kapitel 4. Die klassifikatorische Bedeutung des Kompositums erlaubt durchaus, weil das Erstelement ja nur ein Subklassenindex ist, ,abweichende“ syntaktische Modifikation: Der Madiran ist ein tief schwarzer Rotwein; ein feiner Grobschmied etc. Regelhaft und systematisch findet sich klassifikatorische Modifikation auch bei Phrasen, wenn sie aus einem Relationsadjektiv und einem Substantiv bestehen (ibd: 47 - und dann ausführlich in Kapitel 6).

Den Prototyp der nicht-klassifikatorischen Komposita fasst die Autorin (Kapitel 4.4) als „Textfunktionskomposita“ (ibd.: 67) zusammen. In der Literatur heißen sie auch Augenblicks-, Textoder Ad-hoc-Komposita. Sie dienen der Textreferenz, der textuellen Informationsverdichtung, und haben keine Affinität zum Lexikon. Als typische nicht-klassifikatorische A+N-Komposita wertet die Autorin Bildungen, die in der älteren Literatur zum Adjektivattribut wohl überwiegend zu den ,unlogischen“ Attributen gerechnet worden wären, die nämlich, in denen die Gültigkeit des Kopfnomens relativiert oder in Frage gestellt wird: angeblich, mutmaßlich, vermeintlich, zukünftig etc. Die nun kommen, ihrer morphologischen Komplexität wegen, in $\mathrm{A}+\mathrm{N}-$ Komposita nicht vor (cf. ibd: 79), aber die Autorin gibt Beispiele wie Fiktiv-Regisseur, auch Falschgeld und ähnliche Bildungen. Weiterhin gelten Komposita mit referenzspezifizierenden Adjektiven als Kandidaten für nicht-klassifikatorische Bildungen. In der Diskussion (Kapitel 4.4.3) werden die Schwierigkeiten der Grenzziehung zwischen klassifikatorischen und nicht-klassifikatorischen Bildungen thematisiert.

Das folgende Kapitel 5 handelt von den semantischen Relationen zwischen Grundwort und Bestimmungswort in der $\mathrm{N}+\mathrm{N}-$ und in der $\mathrm{A}+\mathrm{N}-$ Komposition. Barbara Schlücker geht der These nach, dass beide sich in puncto semantische Relationen sehr grundlegend unterscheiden. Wegen der kategorialen Modifikatorbedeutung von Adjektiven bezweifelt die Autorin (ibd.: 


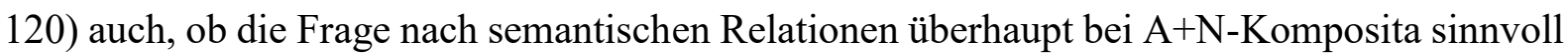
gestellt werden kann. Ihre Untergruppe 1 umfasst dann die Bildungen, die sich einzig durch den klassifikatorischen Zusatzakzent von den entsprechenden Phrasen unterscheiden (Altstadt, Billigflug, Bitterstoff, Hartkäse, Heißgetränk etc.). Die Gruppe 2 umfasst Possessivkomposita mit adjektivischem Erstglied (Blauhelm, Braunhemd, Blondschopf etc.), bei denen man von einer externen Possessum-Possessor-Relation ausgehen kann: Der Possessor ist das vom inalienablen Possessum her indizierte Konzept, zu dem der avisierte Referent sortal gehört. Die 3. Gruppe schließlich bilden Komposita, deren Erstelement als Adverb zu einem ausgelassenen Verb oder Partizip interpretiert werden kann (Scharfschütze, Rundbrief, Schnellimbiss etc.). Das Phänomen der ,,ausgelassenen Partizipien“ ist (dem Rezensenten) vertraut aus der Untersuchung von A+N-Phrasen mit ,agentiven“ Relationsadjektiven vom Typ staatlich, ärztlich, gerichtlich, bei denen ein Großteil (!) der Verwendungen aus substantivierten Adverbialsyntagmen besteht (staatliche Förderung, Unterstützung, Subventionierung..), und ein anderer Teil aus potentiellen Objekten solcher (dann weggelassenen) Verben (staatliche Museen, Labors, Fonds, Schulen, Reiseleiter, Ämter - bis hin zu staatlichem Heroin im Sinne von staatlich verabfolgtem Heroin).

Insgesamt scheint es sich in der Tat, wo eine solche Deutung möglich ist, bei den A+N-Komposita um ganz andere ,semantische Relationen“ zu handeln als im Bereich der N+N-Komposita - aber auch da kann man den theoretischen Nutzen solcher Relationslisten (ibd.: 114f.) bezweifeln. Sie improvisieren semantische Ordnung für ein Feld, für das (nach einer beliebten Formulierung) gilt: N1 + N2-Kompostita stehen für ein N2, das irgendetwas mit N1 zu tun hat. Semantische Relationen fassen bestenfalls zusammen, wie sich diese Beziehbarkeiten extrakommunikativ ordnen lassen.

Kapitel 6 handelt dann von lexikalischen A+N-Phrasen im Deutschen und arbeitet heraus, dass es in der Hauptsache zwei Unterschiede gegenüber den Komposita gibt: Einmal haben A+NPhrasen typischerweise sowohl eine klassifikatorische als auch eine (weniger häufige, aber abrufbare) deskriptiv-charakterisierende Lesart (gelbe Karte, blauer Fleck, blauer Brief etc.), zum anderen überwiegen in dieser Abteilung „,metaphorische Köpfe“ (altes Eisen, dicke Luft, heißer Draht, hohes Tier, kalter Kaffee) und/oder Onymika (Weißes Haus, Rotes Kreuz, Gelbe Seiten), die übrigens auch meist eine zur Not abrufbare einfach charakterisierende Lesart haben.

Im (recht knappen und etwas tastenden) Kapitel 7 geht es dann um die begriffliche Bestimmung von „Benennungseinheiten“, die ja, wie gesagt, von klassifikatorischen Lexemen unterschieden werden müssen und sowohl lexikalisch als auch phrasal sein können. Auch ein Textkompositum wie Kachelmann-Prozess ist eine Benennungseinheit, ebenso wie A+N-Phrasen des Typs rechter Winkel, grüne Welle, wilde Ehe (ibd: 191). Hilfreich für dieses Kapitel wäre möglicherweise das Buch von Pavlov (2009) gewesen, der dieser Frage (übrigens auch an vielen nennfesten A+N-Phrasen wie öffentliche Meinung, letzter Wille, frische Luft, alkoholfreies Bier) auch diachron nachgeht. Auch für ihn pendeln die Komposita zwischen lexikalischer Reproduzierbarkeit auf der einen, textueller Zusammenfassung auf der anderen Seite. Und sein „Feldstrukturmodell“: Jedes Formschema (heute würde man wohl „Konstruktion“ sagen) steht für ein Bündel von Funktionen, und für jede Funktion gibt es ein geordnetes Bündel von Formschemata (ibd.: 22) wäre durchaus anschlussfähig für das Theoriekonzept der Autorin. 
Das 8. Kapitel macht den Übergang zu einer psycholinguistischen Produktionsstudie, die in ausführlicherer Form separat veröffentlicht ist (cf. Schlücker/Plag 2011). Geprüft wird die Frage: Welche Faktoren beeinflussen, ob eine neue A+N-Einheit eher als Phrase oder eher als Kompositum gebildet wird. Während Strukturlinguisten gerne annehmen, dass der Kopf die selektionsentscheidende Instanz ist, gibt es in der Psycholinguistik (verbunden mit dem Namen von Christina Gagné) recht starke (empirische) Argumente dafür, dass eher der Modifikator die analogische Bahnung zu beeinflussen vermag (aber natürlich nicht nur der Modifikator). Etwas vereinfacht: Das Adjektiv sauer kommt ungefähr ebenso oft in klassifizierenden A+N-Phrasen (saure Sahne, saure Gurken, saure Heringe etc.) wie in A+N-Komposita vor (Sauerkirsche, Sauerbraten, Sauerampfer, Sauermilch etc.). Je ungleicher eine solche Verteilung ausfällt, je stärker das Übergewicht eines Musters, desto größer die Wahrscheinlichkeit, dass (per familysize-Effekt) Neubildungen dem stärkeren Muster folgen. Auch die Köpfe zeigen solche Präferenzen (Milch neigt zu Komposita: Dickmilch, Magermilch, Vollmilch, Frischmilch, Rohmilch etc.). Die Neubildungen basieren also auf Netzen multipler Analogien und Bahnungen, und das Experiment zeigt, dass für Sprecher $\mathrm{A}+\mathrm{N}-\mathrm{Phrasen}$ und $\mathrm{A}+\mathrm{N}-\mathrm{Komposita}$ konkurrierende Muster sind, zwischen denen gewichtete Analogien entscheiden (wobei einem Sprachwissenschaftshistoriker die Flexibilität des Analogiebegriffes ein wenig unheimlich ist!).

Kapitel 9 schließlich bereichert die Evidenz durch einen Seitenblick auf die Verhältnisse im Niederländischen, die sich insgesamt als den deutschen Verhältnissen recht ähnlich erweisen, auch wenn es dort vergleichsweise mehr A+N-Phrasen mit Nenneigenschaften gibt. Ein Grund dafür, so die Autorin, könnte darin liegen, dass A+N-Komposita niederländischen Sprachpflegern als Germanismen gelten, die zu vermeiden seien. Kapitel 10 gibt eine knappe und konzise Zusammenfassung der Arbeit.

Theoretisch stützt sich die Untersuchung Barbara Schlückers auf Jackendoffs parallel-architecture-Modell, dessen Axiomatik drei eigenständige, aber miteinander verbundene Strukturebenen vorsieht: phonologische, syntaktische und konzeptuelle Strukturen, die nach der einen Seite als Interface zum sensomotirischen System von Artikulation und Hören, nach der anderen Seite als Interface hin zu Wahrnehmung und Handlung dienen (cf. Schlücker 2014: 6-13). Die Syntax ist in diesem Modell sparsam, sie gilt nicht als Demiurg und eigentliches Kraftzentrum des Systems, sondern als bloße Hilfs- und Vermittlungsgröße für die recht eigenständige konzeptuelle Kombinatorik. Die Autorin stellt sowohl die Affinitäten wie auch die Unterschiede zur (derzeit modischen) Konstruktionsgrammatik heraus: Konstruktionsformate gelten nicht selbst als Zeichen mit systemischer Bedeutung bei Jackendoff. Insgesamt hält Barbara Schlücker gleichermaßen erfreuliche Distanz zum Notationsrealismus vieler generativer Grammatiker wie zum Zahlenfetischismus der wild blühenden Korpusgrammatik. Die Arbeit beruht empirisch auf einer reichen und gründlich analysierten Materialsammlung sowie subsidiär auf der psycholinguistischen Produktionsstudie und dem Vergleich zum Niederländischen.

Das Buch setzt Maßstäbe auf dem Weg zu einer vergleichenden „Noetik“ (Koschmieder 1965: 70-89) phrasaler und lexikalischer Nenneinheiten. Was Bühler (1934) als „Feldbruch“ zwischen Wort und Syntagma bezeichnet (und was in der Sache ungefähr den Beobachtungen entspricht, die heute eher als ,anaphorische Insel“-Eigenschaft von Wortbestandteilen gefasst wird, die „von außen“, aus dem syntagmatischen Umfeld, nicht adressierbar sind), das ist bei weitem noch nicht hinreichend erforscht und verstanden. Ebenso fehlt es an systematischen Erkenntnissen auch über „normale“, d. h. nicht-klassifikatorische A+N-Phrasen, bei denen im 
Prinzip das Adjektiv das Kopfkonzept und/oder dessen Referenzgröße modifizieren kann. Bei den aus verbalem Prädikat und Referenzierungsindex zusammengestellten Nomina agentis auf -er z. B. (Arbeiter, Penner, Läufer, Schläfer...), neigen wir zu dephrasalen, aus der AdverbVerb-Beziehung abgeleiteten konzeptuellen Lesarten, wo diese möglich sind (ein fleißiger, gründlicher, sorgfältiger Arbeiter), wo sie nicht möglich sind, charakterisiert das attributive Adjektiv den indizierten personalen Referenten (ein großer, stämmiger, blonder Arbeiter). Oft sind die Grenzen zu dephrasalen, vor allem auf Adverb-Verb-Beziehungen zurückgehenden Lesarten nur schwer zu ziehen. Aber erkennbar sind die klassifizierenden (oder konzeptmodifizierenden) Bedeutungstypen, um die es in Barbara Schlückers Untersuchung geht, eine Spezialisierung auf der konzeptuellen Achse, die eine neue sortale Einheit definiert.

An einigen Stellen der Untersuchung wäre Anschlussforschung sinnvoll (und vorzüglich angeleitet durch die Ergebnisse der Autorin). Nur einige wenige Beispiele: Eine interessante Ergänzung zu der psycholinguistischen Produktionsstudie (Kapitel 8) könnten Spracherwerbsuntersuchungen liefern. Hall/Moore (1997) z. B. haben Vorschulkinder (und Erwachsene) mit kategorial unterschiedlichen Nenninformationen zu abgebildeten Objekten versorgt, um herauszufinden, ob und wie sie die kategorialen Informationen bei der weiteren Verwendung der Ausdrücke berücksichtigen:

\section{a. $\quad$ This is a blue (Substantivslot) \\ b. This is a blue-one (Adjektivslot) \\ c. This is a blue bird (Kompositumformat, betontes Adjektiv) \\ d. This is a blúe bird (A+N-Phrase, Hauptton auf dem Kopf}

Mit weiteren Referenzangeboten kann man dann überprüfen, ob das Kompositumformat dazu führt, dass ein andersfarbiger, aber typgleicher Vogel der blúe bird - Kategorie zugewiesen wird. Hall/Moore (1997: 236) kommen zu dem Ergebnis, dass ungefähr ab einem Alter von 4;0 „,children treated a word's lexical category, cued syntactically or phonologically, as a powerful cue to its meaning".

Während pscholinguistische Produktionsstudien des oben beschrieben Typs immer ein wenig davon abhängen, dass die Bahnungs- und Häufigkeitsverhältnisse (die family-sizes) im Kopf der Versuchspersonen ungefähr den anderweitig erhobenen Gebrauchsfrequenzen entsprechen, zielen Erwerbsuntersuchung wie die von Hall/Moore (1997) tatsächlich stärker auf den kategorialen Aspekt der Konstruktionen.

Weiterhin stößt die Autorin von Zeit zu Zeit auf Befunde, die den Schluss nahelegen, dass Evaluativa sich im Feld der Komposition grundsätzlich anders verhalten als Konzeptmodifizierer. Sie führen z. B. im Bereich der N+N-Komposita zu Bildungen mit Doppelakzent: Mórdsproblém, Riésenspáß, Bómbenstímmung (Schlücker 2014: 54). In letzter Zeit ist die These vertreten worden, dass es nicht nur eine Unzahl von Adjektiven mit primär evaluativer, bewertender Bedeutung gibt, sondern dass auch die (vermeintlich) anderen und „objektiven“ oder „kognitiven“ Adjektive, sofern sie charakterisierend sind (und keine Relationsadjektive), aktivierbare evaluative Komponenten haben (cf. die Beiträge in Schmale 2011). Ein runder Text, eine steile These, ein eckiges Verhalten, ein schwarzer Tag sind nur ein paar Beispiele. Möglicherweise taugen evaluative Adjektive nur begrenzt zu klassifkatorischen Bildungen, weshalb der Gutmensch eine auffällig und ziemlich einmalige Erscheinung bleibt. 
Und schließlich noch eine letzte Bemerkung zu der zentralen (und, wenn ich recht sehe, sehr konsensfähigen) These, nur monomorphemische Adjektive seien zulässig für die Bildung von A $+\mathrm{N}-$ Komposita. Dass die Beschränkung nicht silbisch sein kann, belegen zahllose Beispiele (Pauschalreise, Direktmandat, Extremfall). Bei den nicht-nativen Ausnahmen auf -al, -iv, -är, -ar fällt immerhin auf, dass alle diese Suffixe auch Substantive bilden (Prinzipal, Stativ, Pensionär, Notar). Schwierig unterzubringen ist auch, dass die (zweifellos zweimorphemigen) Superlative ohne weiteres gehen in der Komposition, und die (gleichermaßen zweimorphemigen) Komparative keinesfalls. Wie wäre es alternativ mit der These, dass der slot des Bestimmungswortes alles, was er aufnimmt auf reine Nennwerte reduziert, auf ein kategorial unmarkiertes, aber der „neutralen“ Substantivklasse am nächsten stehendes Format? Dann wäre er gesperrt für alle Größen, die morphologisch explizit als nicht-substantivisch ausgewiesen sind. Dass Superlative „substantivischer“ sind als die (im Kern prädikativen) Komparative, ließe sich notfalls zeigen!

\section{Literatur}

Bühler, Karl (1934): Sprachtheorie. Die Darstellungsfunktion der Sprache. Jena: Fischer.

Hall, Geoffrey/Moore, Catherine (1997): "Red bluebirds and black greenflies: Children's understanding of the semantics of adjectives and count nouns". In: Journal of Experimental Child Psychology 67: 236-267.

Koschmieder, Erwin (1965): Beiträge zur allgemeinen Syntax. Heidelberg: Winter.

Paul, Hermann (1920): Prinzipien der Sprachgeschichte. 5. Aufl. Tübingen: Niemeyer.

Pavlov, Vladimir (2009): Deutsche Wortbildung im Spannungsfeld zwischen Lexikon und Syntax. Frankfurt a. M., Berlin: Lang.

Schlücker, Barbara/Plag, Ingo (2011): “Compound or phrase? Analogy in naming”. In: Lingua 121/7: 1539-1551.

Schmale, Günter (ed.) (2011): Das Adjektiv im heutigen Deutsch. Syntax, Semantik, Pragmatik. Tübingen: Stauffenburg. 